\title{
CONSTRUCTIVE SIMULATION PROGRAMS AND NATO FUNCTIONAL AREA SERVICES APPLIED IN COMPUTER ASSISTED EXERCISES
}

\author{
Diana-Ioana ZINCA (NEAGOE) \\ Multinational Division South-East Headquarters, Bucharest, Romania \\ zinca_diana@yahoo.com; diana.neagoe@mndse.nato.int \\ Ghiţă BÂRSAN \\ “Nicolae Bălcescu” Land Forces Academy, Sibiu, Romania \\ gbarsan@armyacademy.ro
}

\begin{abstract}
In the military system, the simulation represents the most important tool to support training because it can be efficiently used from the tactical level up to the strategic level. For individual training the costs of using real or virtual simulation systems are not justified, but when it comes to the headquarters training, running a live exercise is extremely expensive. Nevertheless, the units, headquarters, general staff and Multinational Division South-East Headquarters need to be trained in both national and NATO exercises. Taking those facts into consideration, computer assisted exercises proved to be the best solution for training all the cycles within a headquarters that support the decision making process. In the last years, among all those processes, a particular interest was shown for the functional area services interoperability during NATO computer assisted exercises.
\end{abstract}

KEYWORDS: computer assisted exercise, functional area services, constructive simulation

\section{Introduction}

In a military environment that is continuously changing, the power of information becomes critical for mission accomplishment. Military forces must be ready to act in different kind of situations, and commanders must be educated to shorten the time required to make decisions. The link between the guidance given by strategic level leadership and mission accomplishment at the tactical level runs through multiple, well-structured processes leading to achievement of the objectives.
All those processes are supported by systems, specially designed to enable the decision-making process. These systems are the basic tools to compute and manage the databases in order to provide the commander all the information needed about friendly forces and the enemy's capabilities during the battle.

\section{(CAX)}

\section{Computer Assisted Exercises}

The "train as you fight" concept turned out to be the touchstone theme for training 
through simulation. In the military system the simulation is used for training, providing the soldiers the opportunity to increase their knowledge, to rehearse their skills during war gaming and to optimize the decision making process within their organization. Whether we talk about testing programs' new versions, users training or personnel integration in the headquarters' processes, the best way to achieve those objectives is by taking part at CAX. These exercises are used in NATO for collective training purposes, including both unit tactical task training and also headquarters processes.

Training through simulation is a new and useful method for training, as it is the less expensive way of collective training and giving the chance to streamline personnel's own skills as well as the organization's decision making process at all three levels (tactical, operational and strategic). CAX are part of this new method and are mostly applied in Headquarters/ General Staff units or in Academic domain. They use "modeling and simulation techniques in order to create an artificial environment, similar to the image of modern battle field, which will stimulate the decision making process and will determine force's future actions" (SMG, 2017, p. 56).

The planning process for this CAX is very well structured and developed. It involves not only the participation of Simulation Centers, but also the implication of trusted agents from training audience units. When it comes to CAX structure, there are two major parts that need to be taken into consideration: Training audiences (Primary Training Audience (PTA) or Secondary Training Audience (STA)) and Exercise Control (EXCON) structures. EXCON is comprised of CIS (Communication and Information Systems), real life support (RLS), Situational Centre (SITCEN) and Response Cells (RC).
Within SITCEN are found the support structures for the exercise as: scenario cells, MEL/MIL (Main Event List/ Main Incidents List) cell, and the CAX support cells who deal with database management and the constructive simulation programs (NATO, 2013,). During the exercise, Response Cells play the higher or the lower echelons role (HICON or LOWCON).

The main objectives for this type of exercise is the simulation of decision making process and the end state that needs to be achieved is the capability to efficiently predict future courses of actions. The systems that we are going to present next in this article are JCATS (Joint Conflict and Tactical Simulation) and JTLS (Joint Theater Level Simulation), the simulation programs used during CAX.

\section{JCATS and JTLS - Constructive Simulation Programs \\ Both JCATS and JTLS are constructive} simulation software used in collective training. JCATS is usually used during exercises for the tactical level, while JTLS supports training at operational and strategic level. Romanian simulation centers are equipped with JCATS system, which brings us to the conclusion that this is the only constructive simulation program that supports the training of Romanian Forces Units. JTLS is used by Multinational Division South-East (MND-SE) Headquarters during NATO exercises. The only simulation center from NATO equipped with JTLS is Joint Warfare Centre located in Stavanger, Norway.

Speaking of JCATS from historical perspective, this simulation system was developed by Lawrence Livermore National Laboratory Company. It was made based on other two simulation programs' architecture: JCM (Joint Conflict Model) and JTC (Joint Tactical Conflict), which were developed in 
United States of America by CSL (Conflict Simulation Laboratory).

"JCATS is a joint high resolution constructive simulation, where the details like a single troop can be simulated by using high resolution terrain and environmental data" (Cayirci, 2007, p. 12-6), designed to support collective training at the tactical and operational level (Welz, 2016, p. 5). This feature allows the user to play either a simple entity or the whole unit, as the exercise requires. Due to the capability of collecting data, JCATS is efficient during the assessment phase of the exercises. To be more specific, the program can be used in Lessons Learned development.

Among its most important features we want to highlight the server-client architecture, the capability to run in batch, the database management and the possibility to link the program with HLA (High Level Architecture), DIS (Distributed Interactive Simulation) and GCCS (Global Command and Control System).

The Romanian Simulation Centers from Cincu-Land Forces Combat Training Center "Getica" and Simulation Training Center from Bucharest use version 13.0 of JCATS and most of their exercises are going up to the brigade level. Multinational Division South-East Headquarters training is supported by this simulation program, the most recent exercise was actually MND-SE CREVAL (Combat Readiness EVALuation of land headquarters and units) exercise, DACIAN LANCER 2018. In order to train this headquarters as lower echelon under JFCNP (Joint Force Command Naples) and as part of NATO organization, JCATS program is not enough. A higher simulation system is required in order to support training at operational and strategic level, and that program is called JTLS.

Joint Theatre Level Simulation-JTLS "is a joint highly aggregated constructive simulation system, which fits operational or higher theatre level simulation requirements" (Cayirci, 2007, p.12-6). Roland \& Associates Corporation Company designed JTLS architecture in order to be able to assess, at macroscopic level, the result of force engagement. From mathematics perspective, JTLS uses Lanchester's laws to calculate combat assessment and for each structure this simulation system runs different types of algorithms. In order to increase exercise's realism, the Common Operational Picture (COP) is accessible to users. JTLS is used for training from division level up and this allows the users to play only with units. The program does not allow us to go down and play with only one entity. This is the main difference between JCATS and JTLS systems. Both of those systems are though used in NATO units training through simulation.

As I mentioned before, Romania does not have this type of equipment. The most recent version of JTLS-version 5.0 is used in the simulation centre from Stavanger. This version of the system was tasted for the first time during TRIDENT JAVELIN 2017 exercise, known as the biggest computer assisted exercise that was ever played in NATO.

\section{Functional Area Services (FAS)}

\section{and Constructive Simulation in} Computer Assisted Exercises

So far in this article were presented the concepts of CAX and constructive simulation programs that support it. Unit training doesn't involve though only simulation systems. The idea to "train as you fight" requires skills training and systems user training. Speaking about NATO structures, our attention is focused on collective training, which means that our training objective is to improve the decision 
making process within a headquarters/ unit. Each person from the organization has to be able to work with systems in order to support C2 (Command and Control). Those standard programs, known in NATO as functional area services (FAS) equip every military unit. The importance of those FASs is emphasized by their databases which can be accessed by all level units. The programs we are talking about run on the NATO SECRET network, but most of them can export their databases, for training purposes, on the MISSION SECRET network. This switch of networks happens because the level of classification for a CAX is usually NATO UNCLASSIFIED, the scenario and all the data from exercise databases are not real and is the proper way to protect the operational database from the training one. In a NATO Headquarters, each structure is trained to use FASs. Those systems are usually interoperable. For instance, the program used the most by the logistic structures is Logistics Functional Area System-LOGFAS (this system is also used by Romanian military units for both operational and training purposes), the intelligence structures use INTEL-FS (Intelligence Functional System), TOPFAS (Tool for Operational Planning, Force Activation and Simulation) and JTS (Joint Targeting System), the planners from each structure work with TOPFAS and TOPFAS-SAT, while the common operational picture is developed in NCOP (NATO Common Operational Picture) system. NCOP program is fed by all FASs inputs and all those information build the common operational picture. As I mentioned before, there is a difference between the training databases and the operational ones. The operational databases are secured and can be modified only by the users who have administration credentials for the systems.
Most users in a unit have read only permissions. The databases for the exercises are built in order to train people to work with the systems. This means that during a CAX every user who will work with FAS has administration credentials and is allowed to update the databases. This highlights the importance of a computer assisted exercise in unit's training.

In order to reach "train as you fight" objective, in computer assisted exercises the actions of lower and higher echelons units are played by the constructive simulation programs. Those programs are able to generate reports which support the training audience (TA) battle rhythm. TA does not have direct access to the simulation system. All that they see and play with are the functional area services. Their purpose is to provide as much information as they can in order to support their commander in decision making process. In order for the exercise to get more realistic, is a must to synchronize the simulation database with the FASs' databases. Another crucial request for a CAX from a systems perspective is the need of interoperability between functional area services. Those programs need to be linked with one another in order to feed NCOP and to build the common operational picture. The events and incidents that occur during an exercise are actually injected to the TA (Training Audience) by the response cells through JEMM (Joint Exercise Management Module).This module is an auxiliary tool which supports the scenario and the simulation programs. An example of the systems' architecture used in a computer assisted exercise is presented in Figure no. 1. Here you can see not only the link between the systems, but also the structures responsible of using each program. 


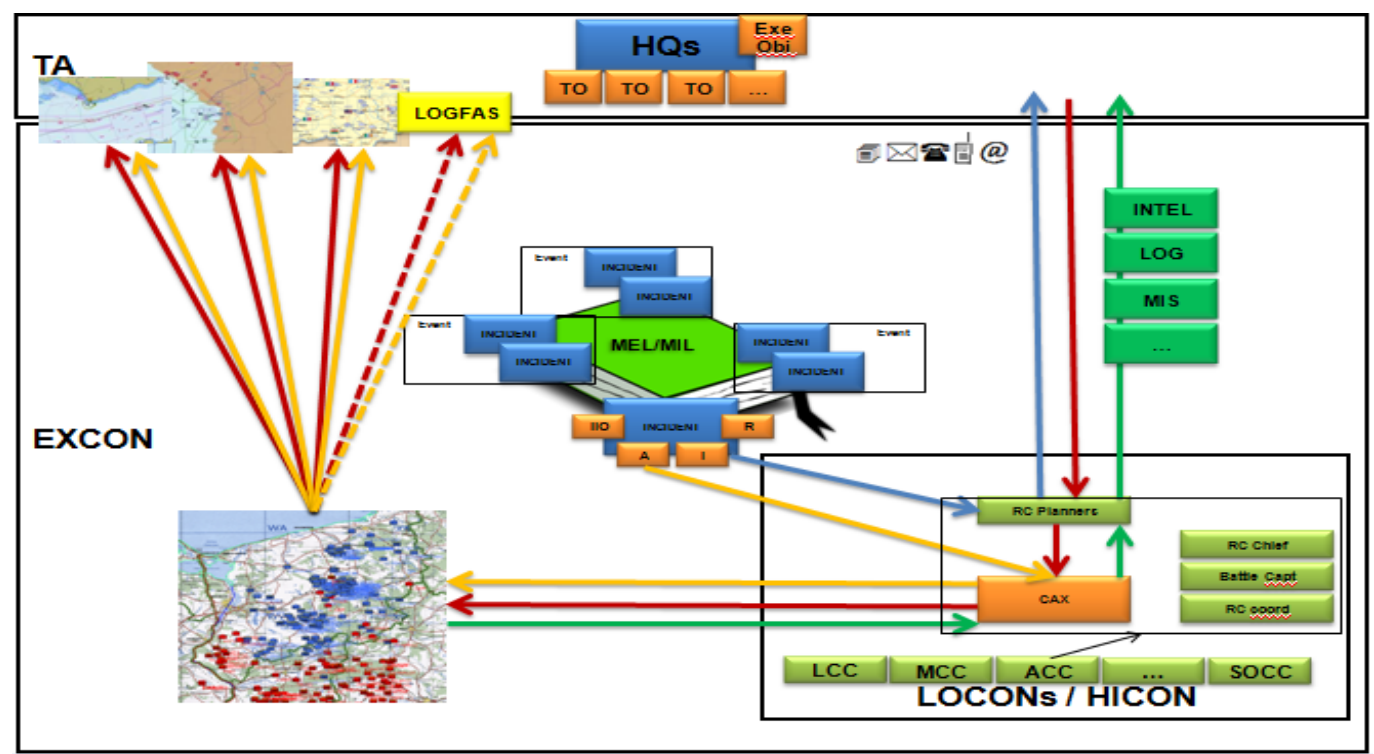

Figure no. 1: Systems' interoperability in a CAX (source: Sacco, 2016, p. 2)

As shown above, TA works with $\mathrm{C} 2$ systems while response cells use JCATS/ JTLS in order to simulate the battlefield, JEMM to inject the events and the FASs to maintain the information workflow within the organization.

To sum up, during a computer assisted exercise two main networks need to be connected. The first one is the simulation network and the second is the operational network supporting TA FASs. When we speak about simulation network we should also take into account DIS (Distributed Interaction Simulation).
Both JCATS and JTLS are a distributed constructive simulation programs. This allows an exercise to be run in different locations. The simulation network is designed in order to connect the main server with the repeater and client servers placed in different locations. Figure no. 2 represents a model of a simulation network. This model was actually used during SABER GUARDIAN 2017 exercise, an exercise that was run in 4 different locations: Bucharest, Cincu, Postonja and Novo Selo.

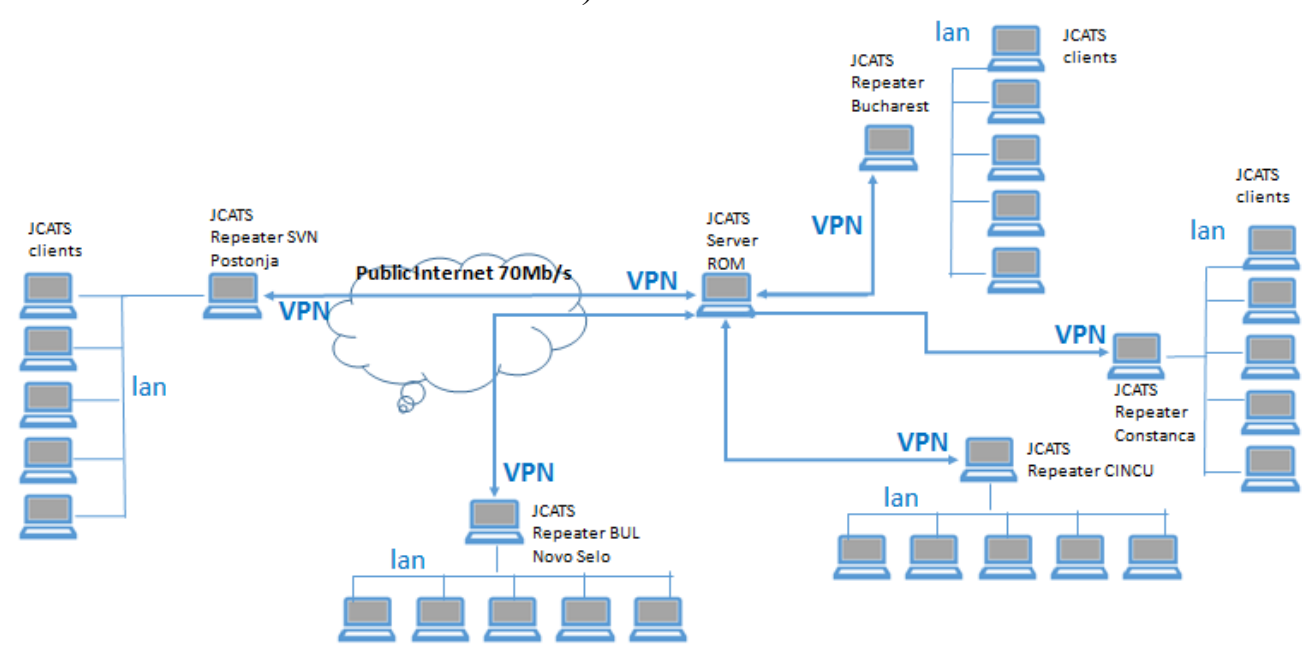

Figure no. 2: Simulation network used during SABER GUARDIAN 2017 (source: Simulation Centre, 2017, p. 2) 
The second type of network within a CAX, the operational network, supports FAS' interoperability. This does not come $100 \%$ into CIS structure responsibility. This is based on each system's capability to connect to another program. Every FAS configuration must allow this link to happen. To achieve that, every system comes with an interface built by the company who developed it. CIS structures with the systems' administrators are only responsible to create bridges to link the FASs. For a better comprehension, please take a look at Figure no. 3. You can see the interface architecture of a functional area service, in this case for JTS. Joint Targeting System is actually the only FAS used in NATO to support the joint targeting cycle. We chose to present you this particular system's interface because in the last two years in NATO was shown a higher interest in optimizing targeting process. Every CAX in NATO at the operational and strategic level had among their training objectives the request to improve joint targeting process. As you can see below, JTS system is capable of interacting with other FASs and is also able to bring its inputs to the common operational picture built in NCOP.

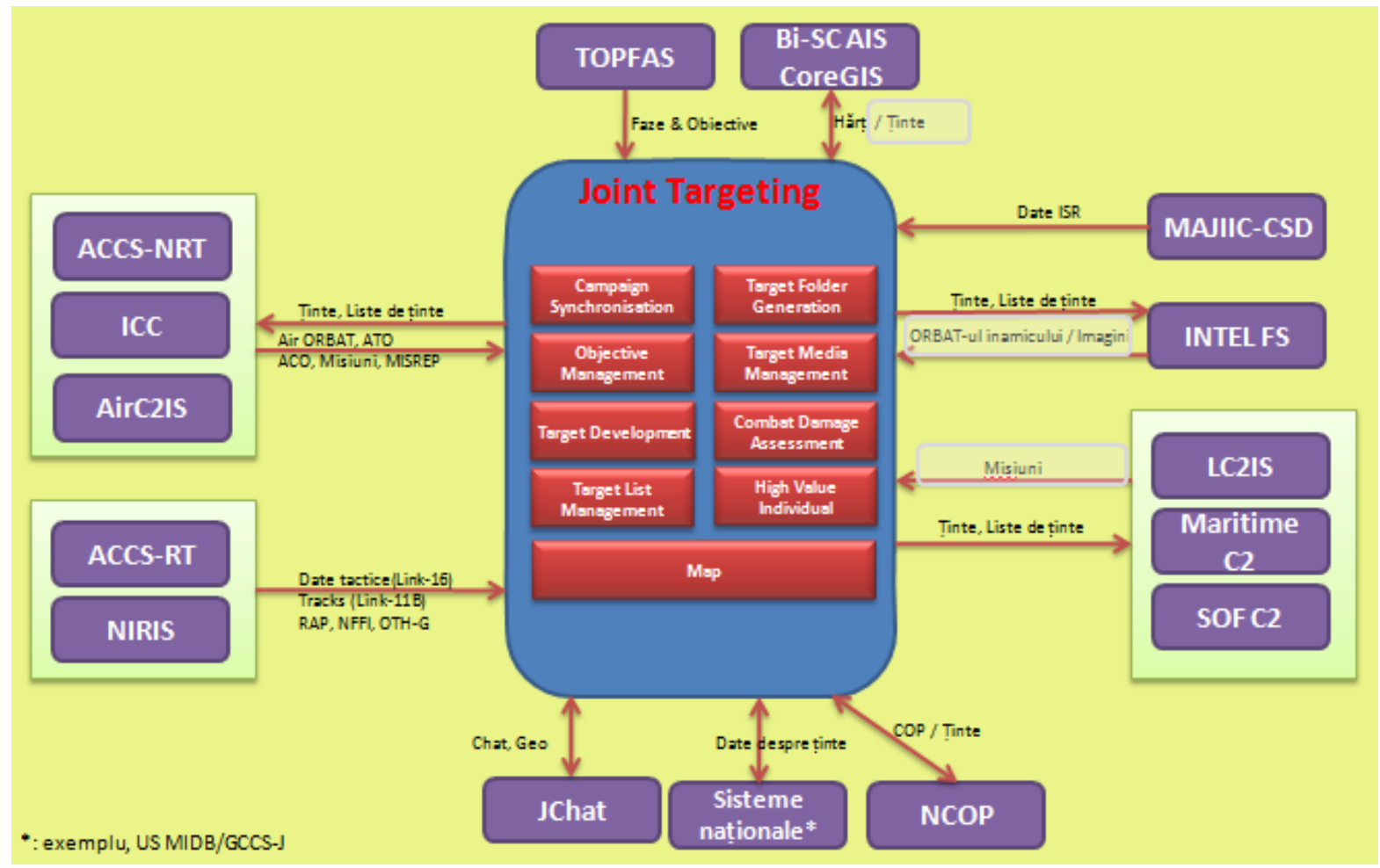

Figure no. 3: JTS v.4.0 system interoperability (source: Xogiannis, 2017, p.7)

\section{Conclusion}

Distributed interactive simulation plays a very important role in computer assisted exercises by defining the simulation network infrastructure in order to allow the exercise to be run in different locations. The simulation systems create a complex, virtual world, mimicking the reality of the battlefield and simulating the unit actions during operations. Constructive simulation programs and NATO functional area services proved their efficiency in decision making process improved the reaction speed of systems users and led the unit to mission accomplishment. Computer assisted exercises are the modern and financially efficient method of training used both by Romania and by NATO. 


\section{REFERENCES}

Cayirci, E. (2007). Multi-Resolution Exercise Control Structure for NATO Education and Training Network, MN-MSG-056-12.

Dimitrios, X. (2017). presentation held during "Advanced FAST FAS \& Dynamic Targeting Training Course JTS-FAST Evolution”, NATO Rapid Deployable Corps-Greece.

NATO. (2013). BI-SC Collective Training and Exercise Directive (CT\&ED) 075-003. Mons, Belgium, Norfolk, USA: Author.

Sacco, L. (2016). Main Events List and Main Incidents List \& JEMM, presentation held during CAX Specialist Course, Rome.

Simulation Centre, proposal for simulation network for the exercise SABER GUARDIAN 2017, available at: https://community.apan.org/ex/saberguardian/ saberstrike18/m/documents/178570;

Statul Major General (SMG). (2017). SMG-10, Ordinul Şefului Statului Major General privind Simularea în Armata Romaniei. Bucureşti: CTEA.

Welz, J. (2016). JCATS Introduction, presentation held during CAX Specialist Course, Rome. 